concentration increased between the two time periods; also contributing to the rise in CKD was an increase in the prevalence of microalbuminuria (albumin:creatinine 30-299 mg/g), from $7.1 \%$ of individuals in $1988-1994$ to $8.2 \%$ in 1999-2004. Increases in the prevalence of diagnosed diabetes and hypertension and in BMI, an aging population, and a decrease in the proportion of non-Hispanic whites all contributed to the observed exacerbation of the CKD problem.

Original article Coresh J et al. (2007) Prevalence of chronic kidney disease in the United States. JAMA 298: 2038-2047

\section{Low-protein diets do not delay progression of chronic kidney disease in children}

Low-protein diets are associated with delayed progression to end-stage renal disease in adults with chronic kidney disease, but there is concern that prescription of such diets to children might lead to impaired growth. A recent Cochrane Review investigated the use of low-protein diets in children.

Searches of databases including MEDLINE and EMBASE identified two trials that enrolled a total of 250 children randomized to either a protein-restricted diet $(0.8-1.4 \mathrm{~g} / \mathrm{kg} /$ day; $n=124)$ or an unrestricted diet $(n=126)$ for $\geq 6$ months. Data on renal death (dialysis commencement, renal transplantation, or patient death) and progression of kidney disease were available for 191 out of 226 patients (aged 2-18 years) who completed $\geq 2$ years of one study. No disease progression was seen in the other study, which enrolled 24 children aged $\leq 6$ months.

The risk of renal death at 2 years was not significantly different between proteinrestricted children and controls (relative risk 1.12, 95\% Cl 0.54-2.33). Creatinine clearance decline was similar between proteinrestricted and control patients at both 2 years and 3 years, and no significant differences were found between groups in severity of proteinuria, systolic blood pressure or diastolic blood pressure at 2 years and 3 years. Height and weight changes were similar in protein-restricted and control patients. Most measures of nutritional status (e.g. upper arm circumference, skin-fold thickness and serum albumin level) were similar in the two groups, although the serum transferrin level was significantly lower in protein-restricted patients $(P=0.006)$.

Original article Chaturvedi $S$ and Jones C. Protein restriction for children with chronic renal failure. Cochrane Database of Systematic Reviews 2007, Issue 4. Art. No.: CD006863. doi:10.1002/14651858.CD006863

\section{Lanthanum carbonate reduces ciprofloxacin bioavailability}

Lanthanum carbonate is a phosphate-binding agent used in people with chronic kidney disease. Little is known, however, about the interaction of this agent with ciprofloxacin, a broad-spectrum antibiotic commonly used to treat infections.

How et al. performed a randomized, openlabel, two-way crossover study to discover whether lanthanum carbonate influences the bioavailability of ciprofloxacin. On each of two consecutive days, six healthy adults received one $750 \mathrm{mg}$ oral dose of ciprofloxacin, plus three $1 \mathrm{~g}$ oral doses of lanthanum carbonate with meals. Another six healthy volunteers received one $750 \mathrm{mg}$ dose of ciprofloxacin on each day but no lanthanum carbonate. All participants crossed over to the other regimen 7-14 days later. The ciprofloxacin concentration in serial blood and urine samples collected during the $24 \mathrm{~h}$ period after the second antibiotic dose was determined by reverse-phase high-performance liquid chromatography.

No differences were observed between the two groups in time to maximum plasma concentration of ciprofloxacin, elimination half-life, or renal clearance. Taking lanthanum carbonate with ciprofloxacin did, however, reduce the antibiotic's systemic availability to $54 \%$ and its maximum plasma concentration to $56 \%$ $(P<0.001)$. These reductions were of greater magnitude than those observed in other studies with different phosphate-binding agents. Only minor adverse events, including nausea, headache and bloating, were reported.

The authors recommend that ciprofloxacin is taken at least $2 \mathrm{~h}$ before or $4 \mathrm{~h}$ after lanthanum carbonate.

Original article How PP et al. (2007) Effects of lanthanum carbonate on the absorption and oral bioavailability of ciprofloxacin. Clin J Am Soc Nephrol 2: 1235-1240 